\title{
REMARKS ON THE BOOLEAN CONVOLUTION AND KEROV'S $\alpha$-TRANSFORMATION
}

\author{
ANNA DOROTA KRYSTEK \\ Mathematical Institute, University of Wrockaw \\ Plac Grunwaldzki 2/4, 50-384 Wrocław, Poland \\ E-mail: Anna.Krystek@math.uni.wroc.pl
}

\begin{abstract}
This paper consists of two parts. The first part is devoted to the study of continuous diagrams and their connections with the boolean convolution. In the second part we investigate the rectangular Young diagrams and respective discrete measures. We recall the definition of Kerov's $\alpha$-transformation of diagrams, define the $\alpha$-transformation of finitely supported discrete measures and generalize the notion of the $\alpha$-transformation.
\end{abstract}

1. Introduction. In the paper [K1] Kerov proved that there a exists $1-1$ correspondence between compactly supported probability measures on the real line and a generalization of Young diagrams, called continuous diagrams. He also studied Young graphs and considered the asymptotics of random growth of Young diagrams and related Markov process. In another paper [K3] Kerov defined a new transformation of Young diagrams, connected with a special dilation on $\mathbb{R}^{2}$. Because of the 1-1 correspondence between finitely supported discrete probability measures on the real line and rectangular Young diagrams, see [K1], we are able to define a corresponding transformation of such measures. We will call it the $\alpha$-transformation.

In Section 2 we recall the necessary notions of rectangular and continuous diagrams along with the definitions of transition and co-transition measures.

In Section 3 we deal with the boolean convolution. The main subject of this section is the study of a correspondence between the boolean convolution and Young diagrams. We define the co-transition measure for continuous diagrams and show that the boolean convolution of compactly supported probability measures coincides with addition of centers of diagrams and addition of co-transition measures.

2000 Mathematics Subject Classification: Primary 46L53, 05E05; Secondary 60J05.

Key words and phrases: interlacing sequences, boolean convolution, Young diagrams. Partially supported by KBN grant no. 2P03A00723 and RTN HPRN-CT-2002-00279. The paper is in final form and no version of it will be published elsewhere. 
In Section 4 we calculate the explicit formulae for the interlacing sequences associated with the $\alpha$-transformed rectangular diagram. Namely, we obtain the following formulae

$$
\begin{aligned}
& x_{k}(\alpha)=\alpha x_{k}+(1-\alpha) \sum_{j=1}^{k-1}\left(y_{j}-x_{j}\right)+(1-\alpha) c, \\
& y_{k}(\alpha)=\alpha y_{k}+(1-\alpha) \sum_{j=1}^{k}\left(y_{j}-x_{j}\right)+(1-\alpha) c,
\end{aligned}
$$

where $c=\sum_{k=1}^{n} x_{k}-\sum_{k=1}^{n-1} y_{k}$.

In Section 5 we extend the definition of the $\alpha$-transformation to discrete finitely supported measures and show that the $\alpha$-transformation commutes with dilation of measures.

In Section 6 we define a generalization of the $\alpha$-transformation, called the $\beta, \alpha$ transformation, and obtain similar results for that new transformation.

2. Interlacing sequences and Young diagrams. We will consider sequences $y_{1}, \ldots$ $\ldots, y_{n-1}$ and $x_{1}, x_{2}, \ldots, x_{n}$. Recall that two sequences are called interlacing if

$$
x_{1}<y_{1}<x_{2}<\ldots<y_{n-1}<x_{n}
$$

and the number

$$
c=\sum_{k=1}^{n} x_{k}-\sum_{k=1}^{n-1} y_{k}
$$

is called the center of interlacing sequences. With every pair of interlacing sequences we can uniquely associate a piecewise linear continuous function $\omega$, called a rectangular Young diagram.

Definition 1. A piecewise linear continuous function $\omega=\omega_{\left(x_{i}, y_{i}\right)}$ is called a rectangular diagram connected with the interlacing sequences $y_{1}, \ldots, y_{n-1}$ and $x_{1}, \ldots, x_{n}$ if

$$
\begin{aligned}
& \omega^{\prime}(u)= \begin{cases}+1 & \text { if } x_{k}<u<y_{k}, \quad k=1,2, \ldots, n, \\
-1 & \text { if } y_{k}<u<x_{k+1}, \quad k=1,2, \ldots, n-1,\end{cases} \\
& \omega(u)=|u-c| \text { if } u<x_{1} \text { or } u>x_{n} .
\end{aligned}
$$

The number

$$
A=\sum_{i<j}\left(y_{i}-x_{i}\right)\left(x_{j}-y_{j-1}\right)
$$

is called the area of the diagram connected with these sequences. We denote the set of such diagrams by $\mathcal{D}_{0}$.

Definition 1 implies that

$$
\begin{aligned}
& \omega\left(x_{k}\right)=\sum_{i=1}^{k-1}\left(y_{i}-x_{i}\right)+\sum_{i=k}^{n-1}\left(x_{i+1}-y_{i}\right), \\
& \omega\left(y_{k}\right)=\sum_{i=1}^{k}\left(y_{i}-x_{i}\right)+\sum_{i=k}^{n-1}\left(x_{i+1}-y_{i}\right),
\end{aligned}
$$


see $[\mathrm{K} 1, \mathrm{~K} 3]$ for proof. The above relations could be rewritten as

$$
\begin{gathered}
\omega\left(x_{k}\right)=c-x_{k}+2 \sum_{j=1}^{k-1}\left(y_{j}-x_{j}\right), \\
\omega\left(y_{k}\right)=c-y_{k}+2 \sum_{j=1}^{k}\left(y_{j}-x_{j}\right) .
\end{gathered}
$$

A true Young diagram $\Lambda=\left(\lambda_{1}, \lambda_{2}, \ldots, \lambda_{m}\right)$, which corresponds to some irreducible representation of the symmetric group $S_{q}$, with $q=\lambda_{1}+\ldots \lambda_{m}$, is uniquely determined by the following conditions:

1. $c=0$,

2. $x_{k} \in \mathbb{Z}$ and $y_{l} \in \mathbb{Z}$ for $k=1,2, \ldots, n$ and $l=1,2, \ldots, n-1$.

We also consider a discrete probability measure

$$
\mu=\sum \mu_{k} \delta_{x_{k}}, \quad \text { where } \quad \mu_{k}=\frac{\prod_{j}\left(x_{k}-y_{j}\right)}{\prod_{j \neq k}\left(x_{k}-x_{j}\right)},
$$

which is called the transition measure of the rectangular diagram $\omega_{\left(x_{i}, y_{i}\right)}$ connected with the interlacing sequences $y_{1}, \ldots, y_{n-1}$ and $x_{1}, \ldots, x_{n}$.

Let $G_{\mu}(z)$ be the Cauchy transform of the measure $\mu$,

$$
G_{\mu}(z)=\sum \frac{\mu_{k}}{z-x_{k}}=\frac{\prod_{j}\left(z-y_{j}\right)}{\prod_{j}\left(z-x_{j}\right)},
$$

and $M_{\mu}(z)$ be the moment generating function of $\mu$,

$$
M_{\mu}(z)=\sum_{j=0}^{\infty} m_{\mu}(j) z^{j}, \quad \text { where } \quad m_{\mu}(j)=\sum x_{k}^{j} \mu_{k}
$$

Then we have

$$
G_{\mu}(z)=\frac{1}{z} M_{\mu}\left(\frac{1}{z}\right) .
$$

Another distribution associated with a pair of interlacing sequences, called the cotransition distribution, arises from the decomposition

$$
\frac{1}{G_{\mu}(z)}=\frac{\left(z-x_{1}\right)\left(z-x_{2}\right) \ldots\left(z-x_{n}\right)}{\left(z-y_{1}\right) \ldots\left(z-y_{n-1}\right)}=z-c-\sum_{k=1}^{n-1} \frac{\nu_{k}}{z-y_{k}},
$$

where $c=c\left(\omega_{\mu}\right)$ is equal to the center of the diagram $\omega$ and $\sum \nu_{k}=A$ equals the area of this diagram [K1]. Moreover

$$
\nu_{k}=-\frac{\prod_{i}\left(y_{k}-x_{i}\right)}{\prod_{i \neq k}\left(y_{k}-y_{i}\right)}
$$

and

$$
x_{1}<y_{1}<x_{2}<\ldots<x_{n-1}<y_{n-1}<x_{n} \quad \Leftrightarrow \quad \nu_{1}, \ldots, \nu_{n-1}>0 .
$$

We are going to consider the following generalization of rectangular diagrams: 
Definition 2. A continuous diagram is any function $\omega: \mathbb{R} \rightarrow \mathbb{R}$ such that

$$
\left|\omega\left(u_{1}\right)-\omega\left(u_{2}\right)\right| \leq\left|u_{1}-u_{2}\right|
$$

and for some $c \in \mathbb{R}$ and sufficiently large $|u|$

$$
\omega(u)=|u-c| .
$$

The number $c$ is called the center of a diagram $\omega$, and the area $A$ of such a diagram is defined as

$$
A=\frac{1}{2} \int_{\mathbb{R}}(\omega(u)-|u-c|) d u .
$$

We denote the set of such diagrams by $\mathcal{D}$.

To every diagram $\omega \in \mathcal{D}$ we associate a probability distribution $\mu=\mu_{\omega}$ which we call the transition distribution of $\omega$. The measure $\mu$ is defined by the identity [K1, K2, K3]

$$
\frac{1}{z} \exp \frac{1}{2} \int_{\mathbb{R}} \frac{d(\omega(x)-|x|)}{x-z}=\int_{\mathbb{R}} \frac{d \mu(x)}{z-x}=G_{\mu}(z),
$$

where $G_{\mu}(z)=\int_{\mathbb{R}} \frac{d \mu(x)}{z-x}$ is the Cauchy transform of the measure $\mu$.

Kerov proved the following theorem:

TheOREM 1 ([K1]). For any diagram $\omega \in \mathcal{D}$ its transition measure exists and is unique and compactly supported. The measure $\mu_{\omega}$ is finitely supported if and only if the diagram $\omega$ is rectangular.

We will also use the following lemma, see [K2] for proof.

LEMMA 2. Let $\mu$ be the transition measure associated with the diagram $\omega$. Let $c$ be the center of $\omega$ and $A$ its area. Then

$$
c=m_{\mu}(1), \quad A=m_{\mu}(2)-\left(m_{\mu}(1)\right)^{2} .
$$

This means that the center of diagram $\omega$ is equal to the mean of its transition measure $\mu$ and the area equals the variance of this measure.

Because the transition measure of continuous diagrams is a generalization of the transition measure of rectangular diagrams, see [K1], we are going to extend the notion of co-transition measure (4) to continuous diagrams. First we recall a lemma proved by Maassen ([Maa], see also $[\mathrm{Ak}]$ ) which characterizes the reciprocals of the Cauchy transforms of measures with finite variance:

Lemma 3. A holomorphic function $F: \mathbb{C}^{+} \rightarrow \mathbb{C}^{+}$is the reciprocal of the Cauchy transform of a measure $\mu$ with finite second moment if and only if there exists a positive finite measure $\rho$ on $\mathbb{R}$ such that

$$
F(z)=z-\alpha_{0}-\int_{\mathbb{R}} \frac{d \rho(x)}{z-x},
$$

where $\alpha_{0} \in \mathbb{R}$ is the first moment of the measure $\mu$.

Using the above lemma, let us define the co-transition measure of continuous diagrams. 
Definition 3. The co-transition measure $\nu$ of continuous diagram $\omega$ is defined by the requirement

$$
\frac{1}{G_{\mu}(z)}=z-c-\int_{\mathbb{R}} \frac{d \nu(x)}{z-x},
$$

where $c$ is a center of $\omega, \mu$ is the transition measure of $\omega$ and $G_{\mu}(z)$ is its Cauchy transform. The co-transition measure of the diagram is positive but not necessarily a probability measure.

3. Boolean convolution. For compactly supported probability measures $\mu, \nu$ their boolean convolution $\mu \uplus \nu$ is defined by the requirement

$$
R_{\mu \uplus \nu}^{B}(z)=R_{\mu}^{B}(z)+R_{\nu}^{B}(z)
$$

where

$$
\frac{1}{G_{\mu}(z)}=z-R_{\mu}^{B}\left(\frac{1}{z}\right)
$$

see $\left[\mathrm{SW}\right.$. The function $R_{\mu}^{B}(z)$ is called the boolean cumulant transform and it can be written as

$$
R_{\mu}^{B}(z)=\sum_{n=1}^{\infty} R_{\mu}^{B}(n) z^{n-1} .
$$

The coefficients $R_{\mu}^{B}(n)$ are called the boolean cumulants. On the level of Cauchy transforms the boolean convolution is equivalent to the formula

$$
\begin{aligned}
\frac{1}{G_{\mu \uplus \nu}(z)} & =\frac{1}{G_{\mu}(z)}+\frac{1}{G_{\nu}(z)}-z, \\
G_{\mu \uplus \nu}(z) & =\frac{G_{\mu}(z) G_{\nu}(z)}{G_{\mu}(z)+G_{\nu}(z)-z G_{\mu}(z) G_{\nu}(z)} .
\end{aligned}
$$

EXAMPLE 1. We are going to calculate the rectangular diagram $\omega$ corresponding to the symmetric two-point measure

$$
\mu=\frac{1}{2}\left(\delta_{-a}+\delta_{a}\right)
$$

We get the following diagram

$$
\omega_{\mu}(x)= \begin{cases}-x & \text { if } x \leq-a \\ x+2 a & \text { if }-a<x \leq 0 \\ -x+2 a & \text { if } 0<x \leq a \\ x & \text { if } a \leq x\end{cases}
$$

Let $\nu$ be also a symmetric two point measure $\nu=\frac{1}{2}\left(\delta_{-b}+\delta_{b}\right)$. Because

$$
G_{\mu}(z)=\frac{z}{(z+a)(z-a)}, \quad G_{\nu}(z)=\frac{z}{(z+b)(z-b)},
$$

for $\mu \uplus \nu$ we have

$$
G_{\mu \uplus \nu}(z)=\frac{1}{2}\left(\frac{1}{z-\sqrt{a^{2}+b^{2}}}+\frac{1}{z+\sqrt{a^{2}+b^{2}}}\right) .
$$


This means that for the boolean convolution $\mu \uplus \nu$ we get the following diagram

$$
\omega_{\mu \uplus \nu}(x)= \begin{cases}-x & \text { if } x \leq-\sqrt{a^{2}+b^{2}}, \\ x+2 \sqrt{a^{2}+b^{2}} & \text { if }-\sqrt{a^{2}+b^{2}}<x \leq 0, \\ -x+2 \sqrt{a^{2}+b^{2}} & \text { if } 0<x \leq \sqrt{a^{2}+b^{2}}, \\ x & \text { if } \sqrt{a^{2}+b^{2}} \leq x .\end{cases}
$$

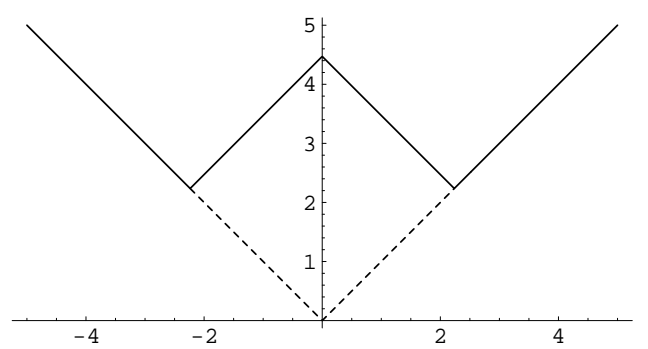

Fig. 1. Diagram corresponding to the $\frac{1}{2}\left(\delta_{-1}+\delta_{1}\right) \uplus \frac{1}{2}\left(\delta_{-2}+\delta_{2}\right)$

Let us also note that the first boolean cumulant $R_{\mu}^{B}(1)$ equals the first moment of the measure $\mu$ and the second cumulant $R_{\mu}^{B}(2)$ is equal to the variance of this measure. This means that from Lemma 2 we get the following corollaries:

Corollary 1. The diagram connected with the boolean convolution $\mu \uplus \nu$ of measures $\mu, \nu$ has center at the point which is equal to the sum of the centers of the diagrams related to the measures $\mu$ and $\nu$.

COROLlary 2. The area of the diagram associated with the measure $\mu \uplus \nu$ is equal to the sum of the areas of the diagrams connected with the measures $\mu$ and $\nu$.

Let $\mu, \rho$ be compactly supported measures. By definition of the co-transition measure (5) we obtain

$$
R_{\mu}^{B}\left(\frac{1}{z}\right)=z-\left(z-m_{\mu}(1)-\int_{\mathbb{R}} \frac{d \nu_{\mu}(x)}{z-x}\right)=m_{\mu}(1)+\int_{\mathbb{R}} \frac{d \nu_{\mu}(x)}{z-x},
$$

or, in the discrete case

$$
R_{\mu}^{B}\left(\frac{1}{z}\right)=c+\sum_{k=1}^{n-1} \frac{\nu_{k}}{z-y_{k}}
$$

This means that

$$
R_{\mu}^{B}(z)=m_{\mu}(1)+\int_{\mathbb{R}} \frac{d \nu_{\mu}(x)}{\frac{1}{z}-x} .
$$

Using the definition of the boolean convolution (6) we obtain

$$
\begin{aligned}
R_{\mu \uplus \rho}^{B}(z) & =R_{\mu}^{B}(z)+R_{\rho}^{B}(z)=m_{\mu}(1)+\int_{\mathbb{R}} \frac{d \nu_{\mu}(x)}{\frac{1}{z}-x}+m_{\rho}(1)+\int_{\mathbb{R}} \frac{d \nu_{\rho}(x)}{\frac{1}{z}-x} \\
& =m_{\mu}(1)+m_{\rho}(1)+\int_{\mathbb{R}} \frac{d \nu_{\mu}(x)+d \nu_{\rho}(x)}{\frac{1}{z}-x}=c_{\mu}+c_{\rho}+\int_{\mathbb{R}} \frac{d \nu_{\mu}(x)+d \nu_{\rho}(x)}{\frac{1}{z}-x} .
\end{aligned}
$$


In terms of the Cauchy transform the above formula is equivalent to

$$
\frac{1}{G_{\mu \uplus \rho}(z)}=z-\left(c_{\mu}+c_{\rho}\right)-\int_{\mathbb{R}} \frac{d\left(\nu_{\mu}(x)+\nu_{\rho}(x)\right)}{z-x} .
$$

Thus we have the following

THEOREM 4. Let $\mu, \rho$ be compactly supported probability measures and let their diagrams have centers at $c_{\mu}, c_{\rho}$ and co-transition measures $\nu_{\mu}, \nu_{\rho}$. Then the diagram related to the measure $\mu \uplus \rho$ has its center at $c_{\mu}+c_{\rho}$ and it is uniquely determined by the co-transition measure $\nu_{\mu}+\nu_{\rho}$.

4. The $\alpha$-transformation of diagrams. In this section we will only consider the rectangular diagrams.

DEFINITION 4. Let $\omega$ be a rectangular diagram connected with the interlacing sequences $y_{1}, y_{2}, \ldots, y_{n-1}$ and $x_{1}, x_{2}, \ldots, x_{n}$. The $\alpha$-transformation $\mathcal{T}_{\alpha}$ of the diagram $\omega$ is the diagram $\omega^{\alpha}$ which is equal to $\omega$ scaled by $\alpha$ along lines which are parallel to the line $y=-x$. The diagram $\omega^{\alpha}$ is connected with the sequences $y_{1}(\alpha), \ldots, y_{n-1}(\alpha)$ and $x_{1}(\alpha), \ldots, x_{n}(\alpha)$, its center is equal to the center of $\omega$ and its area equals $\alpha A$, where $A$ is the area of $\omega$.

REMARK 1. That transformation for true Young diagrams was considered by Kerov in [K3]. He gave formulae for the dimension of $\Lambda^{\alpha}$, called the $\alpha$-hook formula, and showed connections with Jack polynomials and symmetric functions, see [Mac].

First we are going to reformulate the above definition more formally.

LEMMA 5. Let $\omega$ be a rectangular diagram connected with the interlacing sequences $y_{1}, \ldots$, $y_{n-1}$ and $x_{1}, \ldots, x_{n}$ and center in $c$. Then for $(x, y)$ such that $y=\omega(x)$ we obtain

$$
\mathcal{T}_{\alpha}\left(\begin{array}{l}
x \\
y
\end{array}\right)=\frac{1}{2}\left(\begin{array}{c}
(1+\alpha) x+(1-\alpha)(y+c) \\
(1-\alpha)(x-c)+(1+\alpha) y
\end{array}\right) .
$$

Proof. The $\alpha$-transformation can be obtained as the superposition of the rotation of $\omega$ about the center $c$ by the angle $-\frac{\pi}{4}$, the dilation $D_{\alpha}$ for $\alpha>0$ defined as follows

$$
D_{\alpha}(u, v)=(u, \alpha v), \quad(u, v) \in \mathbb{R}^{2},
$$

and the rotation on the center $c$ by the angle $\frac{\pi}{4}$. Hence we get

$$
\begin{aligned}
& \mathcal{T}_{\alpha}\left(\begin{array}{l}
x \\
y
\end{array}\right)=\frac{1}{2}\left(\begin{array}{cc}
1 & -1 \\
1 & 1
\end{array}\right)\left(\begin{array}{ll}
1 & 0 \\
0 & \alpha
\end{array}\right)\left(\begin{array}{cc}
1 & 1 \\
-1 & 1
\end{array}\right)\left(\left(\begin{array}{l}
x \\
y
\end{array}\right)+\left(\begin{array}{c}
-c \\
0
\end{array}\right)\right)+\left(\begin{array}{l}
c \\
0
\end{array}\right)
\end{aligned}
$$

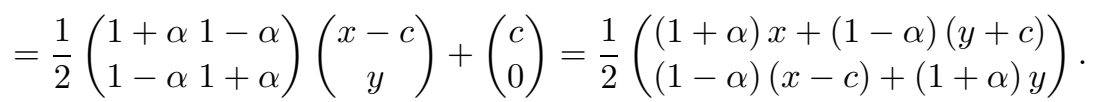

REMARK 2. Let $\omega$ be as in the previous lemma. To calculate the interlacing sequences $y_{1}(\alpha), \ldots, y_{n-1}(\alpha)$ and $x_{1}(\alpha), \ldots, x_{n}(\alpha)$ connected with $\omega^{\alpha}$ let us take $\left(\begin{array}{l}x \\ y\end{array}\right)=\left(\begin{array}{c}x_{k} \\ \omega\left(x_{k}\right)\end{array}\right)$. Then by Lemma 5 we obtain

$$
x_{k}(\alpha)=\frac{1}{2}\left((1+\alpha) x_{k}+(1-\alpha)\left(\omega\left(x_{k}\right)+c\right)\right)
$$


and for $\left(\begin{array}{l}x \\ y\end{array}\right)=\left(\begin{array}{c}y_{k} \\ \omega\left(y_{k}\right)\end{array}\right)$ we get

$$
y_{k}(\alpha)=\frac{1}{2}\left((1+\alpha) y_{k}+(1-\alpha)\left(\omega\left(y_{k}\right)+c\right)\right) .
$$

By the above remark we have a formula for calculating $y_{1}(\alpha), \ldots, y_{n-1}(\alpha)$ and $x_{1}(\alpha), \ldots, x_{n}(\alpha)$ which uses the coordinates $\left(x_{k}, \omega\left(x_{k}\right)\right),\left(y_{j}, \omega\left(y_{j}\right)\right)$. We would like to have also the rule of calculating $y_{1}(\alpha), \ldots, y_{n-1}(\alpha)$ and $x_{1}(\alpha), \ldots, x_{n}(\alpha)$ only in terms of $y_{1}, \ldots, y_{n-1}$ and $x_{1}, \ldots, x_{n}$. The following lemma gives the desired formulae:

LEMMA 6. Let $\omega$ be a rectangular diagram connected with the interlacing sequences $y_{1}, \ldots$, $y_{n-1}$ and $x_{1}, \ldots, x_{n}$ and center at $c$. Then

$$
x_{k}(\alpha)=\alpha x_{k}+(1-\alpha) \sum_{j=1}^{k-1}\left(y_{j}-x_{j}\right)+(1-\alpha) c
$$

and

$$
y_{k}(\alpha)=\alpha y_{k}+(1-\alpha) \sum_{j=1}^{k}\left(y_{j}-x_{j}\right)+(1-\alpha) c .
$$

Proof. By Remark 2 we get

$$
x_{k}(\alpha)=\frac{1}{2}\left(x_{k}+\omega\left(x_{k}\right)+\alpha\left(x_{k}-\omega\left(x_{k}\right)\right)+(1-\alpha) c\right)
$$

and because of (1) we obtain

$$
\begin{aligned}
& x_{k}+\omega\left(x_{k}\right)=c+2 \sum_{j=1}^{k-1}\left(y_{j}-x_{j}\right), \\
& x_{k}-\omega\left(x_{k}\right)=2 x_{k}-c-2 \sum_{j=1}^{k-1}\left(y_{j}-x_{j}\right),
\end{aligned}
$$

which implies

$$
\begin{aligned}
x_{k}(\alpha) & =\frac{1}{2}\left(c+2 \sum_{j=1}^{k-1}\left(y_{j}-x_{j}\right)+\alpha\left(2 x_{k}-c-2 \sum_{j=1}^{k-1}\left(y_{j}-x_{j}\right)\right)+(1-\alpha) c\right) \\
& =\alpha x_{k}+(1-\alpha) \sum_{j=1}^{k-1}\left(y_{j}-x_{j}\right)+(1-\alpha) c .
\end{aligned}
$$

The calculation of $y_{k}(\alpha)$ is similar.

Lemma 7. For $\alpha>0$ the transformation $\mathcal{T}_{\alpha}$ is a multiplicative group:

$$
\mathcal{T}_{\alpha} \mathcal{T}_{\beta}=\mathcal{T}_{\alpha \beta}
$$


Proof. We have

$$
\begin{aligned}
& \mathcal{T}_{\alpha} \mathcal{T}_{\beta}\left(\begin{array}{l}
x \\
y
\end{array}\right)=\mathcal{T}_{\alpha}\left(\frac{1}{2}\left(\begin{array}{c}
(1+\beta) x+(1-\beta)(y+c) \\
(1-\beta)(x-c)+(1+\beta) y
\end{array}\right)\right) \\
& =\frac{1}{2}\left(\begin{array}{c}
1+\alpha 1-\alpha \\
1-\alpha 1+\alpha
\end{array}\right)\left(\frac{1}{2}\left(\begin{array}{c}
(1+\beta) x+(1-\beta)(y+c)-2 c \\
(1-\beta)(x-c)+(1+\beta) y
\end{array}\right)\right)+\left(\begin{array}{l}
c \\
0
\end{array}\right) \\
& =\frac{1}{2}\left(\begin{array}{c}
(1+\alpha \beta) x+(1-\alpha \beta)(y+c) \\
(1-\alpha \beta)(x-c)+(1+\alpha \beta)
\end{array}\right)=\mathcal{T}_{\alpha \beta}\left(\begin{array}{l}
x \\
y
\end{array}\right) \text {. }
\end{aligned}
$$

Hence we obtain

Corollary 3. For $\alpha>0, \mathcal{T}_{\alpha}$ and $\mathcal{T}_{1 / \alpha}$ are inverses of each other.

5. The $\alpha$-transformation of measures. In this section we will only consider finitely supported discrete probability measures.

By the theorem of Kerov, see Theorem 1, we can extend the notion of $\mathcal{T}_{\alpha}$-transformation to a transformation of discrete, finitely supported measures on $\mathbb{R}$.

DEFINITION 5. Let $\mu$ be a finitely supported probability measure and let $\omega$ be the diagram such that the transition measure of $\omega$ is equal to $\mu$. By the $\alpha$-transformation of the measure $\mu$ we mean the transition measure $\mathcal{T}_{\alpha} \mu$ associated with the diagram $\omega^{\alpha}$.

EXAMPLE 2. For a one point measure $\delta_{a}$ we have

$$
\mathcal{T}_{\alpha} \delta_{a}=\delta_{a}
$$

EXAMPLE 3. We compute the $\alpha$-transformation of a probability measure which is supported in two points. Let

$$
\mu=p \delta_{a}+q \delta_{b}, \quad a<b, \quad p, q \geq 0, \quad p+q=1 .
$$

Then we have

$$
x_{1}=a, \quad y_{1}=q a+p b, \quad x_{2}=b, \quad c=p a+q b .
$$

Hence

$$
\begin{aligned}
& x_{1}(\alpha)=\alpha a+(1-\alpha) c, \\
& y_{1}(\alpha)=q a+p b-(1-\alpha)(a-c), \\
& x_{2}(\alpha)=b,
\end{aligned}
$$

and therefore

$$
\mathcal{T}_{\alpha} \mu=\mu_{1} \delta_{x_{1}(\alpha)}+\mu_{2} \delta_{x_{2}(\alpha)},
$$

where

$$
\mu_{1}=\frac{x_{1}(\alpha)-y_{1}(\alpha)}{x_{1}(\alpha)-x_{2}(\alpha)}, \quad \mu_{2}=\frac{x_{2}(\alpha)-y_{1}(\alpha)}{x_{2}(\alpha)-x_{1}(\alpha)}
$$

Thus we obtain

$$
\mathcal{T}_{\alpha} \mu=\frac{p(b-a)}{b-\alpha a-(1-\alpha) c} \delta_{\alpha a+(1-\alpha) c}+\frac{q(b-a)+(a-c)(1-\alpha)}{\alpha a+(1-\alpha) c-b} \delta_{b} .
$$


In general, let $\mu$ be a discrete probability measure supported on $x_{1}, x_{2}, \ldots, x_{n}$, that is,

$$
\mu=\sum_{k=1}^{n} \mu_{k} \delta_{x_{k}}
$$

THEOREM 8. The $\alpha$-transformation of measure $\mu$ is a measure $\mu^{\alpha}$ such that its Cauchy transform is equal to

$$
G_{\mu_{\alpha}}(z)=\frac{\prod_{k=1}^{n-1}\left(z-y_{k}(\alpha)\right)}{\prod_{k=1}^{n}\left(z-x_{k}(\alpha)\right)},
$$

where $x_{k}$ belongs to the support of the measure $\mu$ and $y_{k}$ are zeros of the Cauchy transform of $\mu$ and

$$
\begin{aligned}
& x_{k}(\alpha)=\alpha x_{k}+(1-\alpha) \sum_{j=1}^{k-1}\left(y_{j}-x_{j}\right)+(1-\alpha) c, \\
& y_{k}(\alpha)=\alpha y_{k}+(1-\alpha) \sum_{j=1}^{k}\left(y_{j}-x_{j}\right)+(1-\alpha) c
\end{aligned}
$$

for

$$
c=\sum_{k=1}^{n} x_{k}-\sum_{k=1}^{n-1} y_{k} .
$$

Moreover, we can prove the following lemma connecting the $\alpha$-transformation and the dilation of measures.

Lemma 9. Dilation of measures commutes with the $\alpha$-transformation:

$$
D_{\lambda} \mathcal{T}_{\alpha} \mu=\mathcal{T}_{\alpha} D_{\lambda} \mu \text {. }
$$

Proof. Let us denote the interlacing sequences associated with the measure $\mathcal{T}_{\alpha} D_{\lambda} \mu$ by $\hat{x}_{k}(\alpha)$ and $\hat{y}_{k}(\alpha)$ and sequences connected with the measure $D_{\lambda} \mathcal{T}_{\alpha} \mu$ by $\widehat{x_{k}(\alpha)}$ and $\widehat{y_{k}(\alpha)}$. Because

$$
D_{\lambda} \mu(A)=\mu\left(\lambda^{-1} A\right)
$$

for $\lambda>0$ we obtain

$$
G_{D_{\lambda} \mu}(z)=\int_{-\infty}^{\infty} \frac{1}{z-x} d\left(D_{\lambda} \mu\right)(x)=\sum_{k=1}^{n} \frac{\mu_{k}}{z-\lambda x_{k}}=\frac{1}{\lambda} G_{\mu}\left(\frac{z}{\lambda}\right)=\frac{1}{\lambda} \frac{Q\left(\frac{z}{\lambda}\right)}{P\left(\frac{z}{\lambda}\right)} .
$$

This means that dilation changes the sequences $x_{1}, \ldots, x_{n}$ and $y_{1}, \ldots, y_{n-1}$ into $\hat{x}_{1}, \ldots, \hat{x}_{n}$ and $\hat{y}_{1}, \ldots, \hat{y}_{n-1}$, where

$$
\hat{x}_{k}=\lambda x_{k}, \quad \hat{y}_{k}=\lambda y_{k},
$$

and

$$
c_{D_{\lambda} \mu}=\lambda c_{\mu}
$$


Therefore after the $\alpha$-transformation we obtain

$$
\begin{aligned}
\hat{x}_{k}(\alpha) & =\alpha \lambda x_{k}+(1-\alpha) \lambda \sum_{j=1}^{k-1}\left(y_{j}-x_{j}\right)+(1-\alpha) c_{D_{\lambda} \mu} \\
& =\alpha \lambda x_{k}+(1-\alpha) \lambda \sum_{j=1}^{k-1}\left(y_{j}-x_{j}\right)+\lambda(1-\alpha) c_{\mu}=\lambda x_{k}, \\
\hat{y}_{k}(\alpha) & =\alpha \lambda y_{k}+(1-\alpha) \lambda \sum_{j=1}^{k}\left(y_{j}-x_{j}\right)+(1-\alpha) c_{D_{\lambda} \mu} \\
& =\alpha \lambda y_{k}+(1-\alpha) \lambda \sum_{j=1}^{k}\left(y_{j}-x_{j}\right)+\lambda(1-\alpha) c_{\mu}=\lambda y_{k}
\end{aligned}
$$

and

$$
G_{\mathcal{T}_{\alpha} D_{\lambda} \mu}(z)=\frac{1}{\lambda} \frac{\prod_{k=1}^{n-1}\left(z-\widetilde{y}_{k}(\alpha)\right)}{\prod_{k=1}^{n}\left(z-\widetilde{x}_{k}(\alpha)\right)}
$$

On the other hand

$$
G_{D_{\lambda} \mathcal{T}_{\alpha} \mu}(z)=\frac{1}{\lambda} G_{\mathcal{T}_{\alpha} \mu}\left(\frac{z}{\lambda}\right)=\frac{1}{\lambda} \frac{\prod_{k=1}^{n-1}\left(\frac{z}{\lambda}-y_{k}(\alpha)\right)}{\prod_{k=1}^{n}\left(\frac{z}{\lambda}-x_{k}(\alpha)\right)}=\frac{\prod_{k=1}^{n-1}\left(z-\lambda y_{k}(\alpha)\right)}{\prod_{k=1}^{n}\left(z-\lambda y_{k}(\alpha)\right)}
$$

which means that

$$
\widehat{y_{k}(\alpha)}=\lambda y_{k}(\alpha), \quad \widehat{x_{k}(\alpha)}=\lambda x_{k}(\alpha)
$$

so dilations and $\alpha$-transformations commute.

6. Generalization of the $\alpha$-transformation. The $\alpha$-transformation of diagrams defined in Section 4 consists in scaling the diagram by $\alpha$ along lines which are parallel to the line $y=-x$. We generalize this transformation and introduce a new transformation, which consists in scaling the diagram by $\alpha$ along lines which are parallel to the line $y=-x$ and scaling by $\beta$ along lines which are parallel to the line $y=x$.

DEFINITION 6. Let $\omega$ be a rectangular diagram connected with the interlacing sequences $y_{1}, \ldots, y_{n-1}$ and $x_{1}, \ldots, x_{n}$. The $\beta, \alpha$-transformation $\mathcal{T}_{\beta, \alpha}$ of the diagram $\omega$ is the diagram $\omega^{\beta, \alpha}$, which is equal to $\omega$ scaled by $\alpha$ along lines which are parallel to the line $y=-x$ and is scaled by $\beta$ along lines which are parallel to the line $y=x$. The sequences associated with the diagram $\omega^{\beta, \alpha}$ will be denoted by $\tilde{y}_{1}, \tilde{y}_{2}, \ldots, \tilde{y}_{n-1}$ and $\tilde{x}_{1}, \tilde{x}_{2}, \ldots, \tilde{x}_{n}$. The center of the diagram $\omega^{\beta, \alpha}$ is equal to the center of $\omega$ and its area equals $\alpha \beta A$, where $A$ is the area of $\omega$.

It is possible to find the numbers $\tilde{y}_{1}, \tilde{y}_{2}, \ldots, \tilde{y}_{n-1}$ and $\tilde{x}_{1}, \tilde{x}_{2}, \ldots, \tilde{x}_{n}$ explicitly. First we are going to prove the following

LEMMA 10. Let $\omega$ be a rectangular diagram connected with the interlacing sequences $y_{1}, \ldots, y_{n-1}$ and $x_{1}, \ldots, x_{n}$ and center in $c$. Then for $(x, y)=(x, \omega(x))$ we have

$$
\mathcal{T}_{\beta, \alpha}\left(\begin{array}{l}
x \\
y
\end{array}\right)=\frac{1}{2}\left(\begin{array}{c}
(\beta+\alpha)(x-c)+(\beta-\alpha) y+2 c \\
(\beta-\alpha)(x-c)+(\beta+\alpha) y
\end{array}\right) .
$$


Proof. The $\beta, \alpha$-transformation can be obtained as the superposition of the rotation of diagram $\omega$ about the center $c$ by the angle $-\frac{\pi}{4}$, the dilation $D_{\beta, \alpha}$, where for $\alpha, \beta>0$

$$
D_{\beta, \alpha}(u, v)=(\beta u, \alpha v), \quad(u, v) \in \mathbb{R}^{2},
$$

and the rotation about the center $c$ by the angle $\frac{\pi}{4}$. Hence we get

$$
\begin{aligned}
\mathcal{T}_{\beta, \alpha}\left(\begin{array}{l}
x \\
y
\end{array}\right) & =\frac{1}{2}\left(\begin{array}{cc}
1 & -1 \\
1 & 1
\end{array}\right)\left(\begin{array}{ll}
\beta & 0 \\
0 & \alpha
\end{array}\right)\left(\begin{array}{cc}
1 & 1 \\
-1 & 1
\end{array}\right)\left(\left(\begin{array}{l}
x \\
y
\end{array}\right)+\left(\begin{array}{c}
-c \\
0
\end{array}\right)\right)+\left(\begin{array}{l}
c \\
0
\end{array}\right) \\
& =\frac{1}{2}\left(\begin{array}{c}
\beta+\alpha \beta-\alpha \\
\beta-\alpha \beta+\alpha
\end{array}\right)\left(\begin{array}{c}
x-c \\
y
\end{array}\right)+\left(\begin{array}{l}
c \\
0
\end{array}\right) \\
& =\frac{1}{2}\left(\begin{array}{c}
(\beta+\alpha)(x-c)+(\beta-\alpha) y+2 c \\
(\beta-\alpha)(x-c)+(\beta+\alpha) y
\end{array}\right) .
\end{aligned}
$$

LEMMA 11. For the diagram $\omega$ which is connected with the interlacing sequences $y_{1}, \ldots$, $y_{n-1}$ and $x_{1}, x_{2}, \ldots, x_{n}$ and has center at $c$ we obtain

$$
\begin{aligned}
& \tilde{x}_{k}=\alpha x_{k}+(\beta-\alpha) \sum_{j=1}^{k-1}\left(y_{j}-x_{j}\right)+(1-\alpha) c, \\
& \tilde{y}_{k}=\alpha y_{k}+(\beta-\alpha) \sum_{j=1}^{k}\left(y_{j}-x_{j}\right)+(1-\alpha) c .
\end{aligned}
$$

Proof. By Remark 3 we get

$$
\tilde{x}_{k}=\frac{1}{2}\left(\beta\left(x_{k}+\omega\left(x_{k}\right)\right)+\alpha\left(x_{k}-\omega\left(x_{k}\right)\right)+(2-\alpha-\beta) c\right)
$$

and because of (1) we get

$$
\begin{aligned}
\tilde{x}_{k} & =\frac{1}{2}\left(\beta\left(c+2 \sum_{j=1}^{k-1}\left(y_{j}-x_{j}\right)\right)+\alpha\left(2 x_{k}-c-2 \sum_{j=1}^{k-1}\left(y_{j}-x_{j}\right)\right)+(2-\alpha-\beta) c\right) \\
& =\alpha x_{k}+(\beta-\alpha) \sum_{j=1}^{k-1}\left(y_{j}-x_{j}\right)+(1-\alpha) c .
\end{aligned}
$$

In a similar way we obtain the explicit formula for $\tilde{y}_{k}$.

REMARK 3. Let $\omega$ be as in the previous lemma. For the specific choice of $\left(\begin{array}{c}x \\ \omega(x)\end{array}\right)$ by Lemma 10 we obtain

$$
\begin{aligned}
& \tilde{x}_{k}=\frac{1}{2}\left((\beta+\alpha)\left(x_{k}-c\right)+(\beta-\alpha) \omega\left(x_{k}\right)\right)+c, \\
& \tilde{y}_{k}=\frac{1}{2}\left((\beta+\alpha)\left(y_{k}-c\right)+(\beta-\alpha) \omega\left(y_{k}\right)\right)+c .
\end{aligned}
$$

Lemma 12. For $\beta, \alpha>0, \mathcal{T}_{\beta, \alpha}$ is a multiplicative group:

$$
\mathcal{T}_{\beta_{1}, \alpha_{1}}\left(\mathcal{T}_{\beta_{2}, \alpha_{2}}\right)=\mathcal{T}_{\beta_{1} \beta_{2}, \alpha_{1} \alpha_{2}} .
$$

Proof. The calculations are similar to the $\alpha$-transformation case.

Corollary 4. For $\alpha, \beta>0, \mathcal{T}_{\beta, \alpha}$ and $\mathcal{T}_{1 / \beta, 1 / \alpha}$ are inverses of each other.

We can also define the $\mathcal{T}_{\beta, \alpha}$-transformation of discrete, finitely supported measures on $\mathbb{R}$. 
DEFINITION 7. Let $\mu$ be a finitely supported probability measure and let $\omega$ be the diagram such that the transition measure of $\omega$ is equal to $\mu$. By the $\beta, \alpha$-transformation of the measure $\mu$ we mean the transition measure $\mathcal{T}_{\beta, \alpha} \mu$ associated with the diagram $\omega^{\beta, \alpha}$.

EXAMPLE 4 . For a one point measure $\delta_{a}$ we have

$$
\mathcal{T}_{\beta, \alpha} \delta_{a}=\delta_{a} .
$$

EXAMPLE 5. We compute the $\beta, \alpha$-transformation of a probability measure which is supported on two points. Let

$$
\mu=p \delta_{a}+q \delta_{b}, \quad a<b, \quad p, q \geq 0, \quad p+q=1 .
$$

Then we have

$$
x_{1}=a, \quad y_{1}=q a+p b, \quad x_{2}=b, \quad c=p a+q b .
$$

Hence

$$
\begin{aligned}
& \tilde{x}_{1}=\alpha a+(1-\alpha) c \\
& \tilde{y}_{1}=\beta(q a+p b)+(\beta-\alpha) a+(1-\alpha) c \\
& \tilde{x}_{2}=b
\end{aligned}
$$

which means that

$$
\mathcal{T}_{\beta, \alpha} \mu=\mu_{1} \delta_{\tilde{x}_{1}}+\mu_{2} \delta_{\tilde{x}_{2}}
$$

where

$$
\mu_{1}=\frac{\tilde{x}_{1}-\tilde{y}_{1}}{\tilde{x}_{1}-\tilde{x}_{2}}, \quad \mu_{2} \quad=\frac{\tilde{x}_{2}-\tilde{y}_{1}}{\tilde{x}_{2}-\tilde{x}_{1}}
$$

and we obtain

$$
\mathcal{T}_{\beta, \alpha} \mu=\frac{2 \alpha a-\beta q a-\beta p b-a \beta}{b-\alpha a-(1-\alpha) c} \delta_{\alpha a+(1-\alpha) c}+\frac{b-\beta q a-\beta p b-a \beta+\alpha a+c \alpha-c}{b-\alpha a-(1-\alpha) c} \delta_{b} .
$$

For a discrete probability $\mu$ measure supported on $x_{1}, \ldots, x_{n}$, namely $\mu=\sum_{k=1}^{n} \mu_{k} \delta_{x_{k}}$, we have

THEOREM 13. The $\beta$, a-transformation of discrete measure $\mu$ is a measure $\mu_{\beta, \alpha}$ with the Cauchy transform equal to

$$
G_{\mu_{\beta, \alpha}}(z)=\frac{\prod_{k=1}^{n-1}\left(z-\tilde{y}_{k}\right)}{\prod_{k=1}^{n}\left(z-\tilde{x}_{k}\right)}
$$

where $x_{k}$ belongs to the support of the measure $\mu, y_{k}$ are zeros of the Cauchy transform of $\mu$ and

$$
\begin{aligned}
& \tilde{x}_{k}=\alpha x_{k}+(\beta-\alpha) \sum_{j=1}^{k-1}\left(y_{j}-x_{j}\right)+(1-\alpha) c \\
& \tilde{y}_{k}=\alpha y_{k}+(\beta-\alpha) \sum_{j=1}^{k}\left(y_{j}-x_{j}\right)+(1-\alpha) c
\end{aligned}
$$

for $c=\sum x_{k}-\sum y_{k}$.

Moreover we can prove a result similar to Lemma 9: 
Lemma 14. Dilations of measures commute with $\mathcal{T}_{\beta, \alpha}$ :

$$
D_{\lambda} \mathcal{T}_{\beta, \alpha} \mu=\mathcal{T}_{\beta, \alpha} D_{\lambda} \mu \text {. }
$$

Proof. Similar to the $\alpha$-transformation case.

\section{References}

[Ak] N. I. Akhiezer, The Classical Moment Problem, Oliver and Boyd, Edinburgh and London, 1965.

[K1] S. V. Kerov, Asymptotic Representation Theory of the Symmmetric Group and its Applications in Analysis, Translations of Mathematical Monographs 219, American Mathematical Society, Providence, RI, 2003.

[K2] S. V. Kerov, A differential model for the growth of Young diagrams, Proceedings of the St. Petersburg Mathematical Society 4, 111-130 (in Russian), Amer. Math. Soc. Transl. Ser. 2, 188, Amer. Math. Soc., Providence, RI, 1999.

[K3] S. V. Kerov, Anisotropic Young diagrams and Jack symmetric functions, Funct. Anal. Appl. 34 (2000), 41-51.

[Maa] H. Maassen, Addition of freely independent random variables, J. Funct. Anal. 106 (1992), 409-438.

[Mac] I. G. Macdonald, Symmetric Functions and Hall Polynomials, Oxford University Press, 1995.

[SW] R. Speicher and R. Woroudi, Boolean convolution, in: Free Probability Theory, D. V. Voiculescu (ed.), Fields Inst. Commun. 12, Amer. Math. Soc., 1997, 267-280. 